\title{
Optimizing Product Development Projects under Asynchronous and Aperiodic System-Local Interactions
}

\author{
Masaki Ogura $^{1}$, Masako Kishida ${ }^{2}$, and Ali Yassine ${ }^{3}$ \\ ${ }^{1}$ Division of Information Science, Nara Institute of Science and Technology, Ikoma, \\ Japan \\ ${ }^{2}$ Principles of Informatics Research Division, National Institute of Informatics, Tokyo, \\ Japan \\ ${ }^{3}$ Department of Industrial Engineering \& Management, American University of Beirut, \\ Beirut, Lebanon
}

\begin{abstract}
Project managers are continuously under pressure to shorten product development durations. One practical approach for reducing the project duration is lessening dependencies between different development components and teams. In this paper, we present an analytical framework for optimally allocating resources to shorten the lead-time of product development projects having a time-varying dependency structure. We build our theoretical framework on a linear system model of product development processes, in which system integration and local development teams exchange information asynchronously and aperiodically. By utilizing a convexity result from matrix theory, we show that optimal resource allocation can be efficiently found by solving a convex optimization problem. We provide an illustrative example to demonstrate the proposed framework. This conference paper is a summary of our recent journal paper (Ogura et al., 2019).
\end{abstract}

Keywords: Project management, resource management, resource allocation systems, time/cost/performance trade-offs, project planning

\section{Introduction}

Projects are indispensable and central in most of the industries for performing several types of work (PMI, 2013). In particular, modularization is a widely adopted approach for effective understanding, management, and characterization of complex PD projects. In practice, various technical, physical, and business constraints prohibit us from ignoring dependencies between modules and simply designing each module separately. Therefore, we seek minimizing or eliminating dependencies outside module boundaries (Baldwin and Clark, 2000). Such interdependencies can be reduced by investing in the design rules defining the connections or relationships between modules (Lee et al., 1997). The reduction is achieved through either the creation of a higher level design rule in the design structure matrix (DSM) or internalizing the rule within the design of each module (Baldwin and Clark, 2000).

On the other hand, the current practice for investing in design rules is often based on project managers' intuition or heuristic rules, which may not necessarily lead to the best outcome (Adler et al., 1995; Loch and Terwiesch, 1999). Therefore, the literature described several decision support tools aimed at assisting managers to cost-efficiently invest in design rules 
Part III: Project Management

(see, e.g., Browning and Ramasesh, (2007) and references therein). However, most of the aforementioned decision support tools in the literature rely on an implicit assumption that the PD project architecture is static and, therefore, does not change over time. This assumption is not consistent with reality; for example, information hiding between development teams give rise to a time-varying dependency structure, which causes persistent recurrence of problems in which progress oscillates between being on schedule and falling behind (Yassine et al., 2003).

In this paper, we present a brief summary of our recent publication, in which we present an optimization framework for making a cost-efficient investment in design rules when the underlying dependency structure between modules is changing over time. We illustrate our theoretical framework by focusing on the PD project model with asynchronous interaction between system integration and local development teams (Yassine et al., 2003). By using the stability theory of switched linear systems (Ogura and Martin, 2015) adopted from the systems and control theory, we show that the proposed model is feasible, i.e., the amount of unfinished work converges to zero, if and only if the magnitude of the eigenvalues of a generalized work transformation matrix (WTM) is strictly less than one. We then propose an analytical framework for optimally weakening the dependencies between different product components for accelerating PD projects. We show that the budget-constrained dependency optimization problem (Hartmann and Briskorn, 2010), where we distribute a fixed amount of resource across the project for minimizing its lead-time, can be globally and efficiently solved via convex optimization techniques (Boyd and Vandenberghe, 2004).

\section{Asynchronous and Aperiodic Work Transformation Model}

In this section, we review the asynchronous work transformation model presented by Yassine et al. (2003). We then generalize the model to the case of random and aperiodic information exchange.

In the asynchronous and periodic work transformation model (Yassine et al., 2003), there exist a pair of local and system teams working for the development of a product. The PD process contains $m$ tasks, and each task is separated into development and integration tasks that are performed by the local and system teams, respectively. As in (Yassine et al., 2003), we let $L_{i}(k)$ and $S_{i}(k)$ represent the amount of unfinished work in the $i$ th local and system task at time step $k$, respectively. Let us define the vectors $L(k)=\left[\begin{array}{llll}L_{1}(k) & \cdots & L_{m}(k)\end{array}\right]^{\top}$ and $S(k)=\left[\begin{array}{llll}S_{1}(k) & \cdots & S_{m}(k)\end{array}\right]^{\top}$. If the local and system teams exchange information at every time step, the above variables evolve by the following equation:

$\left[\begin{array}{l}L(k+1) \\ S(k+1)\end{array}\right]=\left[\begin{array}{cc}W_{L} & W_{S L} \\ W_{L S} & W_{S}\end{array}\right]\left[\begin{array}{l}L(k) \\ S(k)\end{array}\right], \quad$ (l)

where $W_{L}, W_{S L}, W_{L S}$, and $W_{S}$ are WTMs having non-negative entries.

As discussed and demonstrated by Yassine et al. (2003), even though the local team may frequently provide the system team with local updates, the system team, on the other hand, may provide only intermittent feedback to the local team. To model this situation, they introduce the third variable, $H_{i}(k)$, denoting the amount of finished work in the $i$ th system task that is not yet transfered to the local team. When feedback from the system to the local team occurs, the finished work will be cleared on the part of the system team and will be 
transferred to the local team. Also, until feedback occurs, the finished work keeps accumulating within the system team. In this situation, the vectorial variable $H(k)=$ $\left[H_{1}(k) \cdots H_{m}(k)\right]^{\top}$ dynamically evolves as

$H(k+1)= \begin{cases}0, & \text { if feedback occurs at time } k, \\ H(k)+W_{S H} S(k), & \text { otherwise, }\end{cases}$

where $W_{S H}$ is a WTM from unfinished system tasks to finished system tasks. Similarly, the amount of unfinished work in the local team evolves by the following difference equation:

$L(k+1)= \begin{cases}W_{L} L(k)+W_{S L} S(k)+H(k), & \text { if feedback occurs at time } k, \\ W_{L} L(k), & \text { otherwise. }\end{cases}$

Finally, notice that the amount of unfinished work in the system team evolves in the same way as in equation (1). Combining the above equations, we see that the joint state variable

$x(k)=\left[\begin{array}{c}L(k) \\ S(k) \\ H(k)\end{array}\right]$

evolves as

$x(k+1)= \begin{cases}A_{1} x(k), & \text { if feedback occurs at time } k, \\ A_{2} x(k), & \text { otherwise }\end{cases}$

where the matrices $A_{1}$ and $A_{2}$ are given by

$A_{1}=\left[\begin{array}{ccc}W_{L} & W_{S L} & I \\ W_{L S} & W_{S} & 0 \\ O & O & O\end{array}\right], A_{2}=\left[\begin{array}{ccc}W_{L} & O & 0 \\ W_{L S} & W_{S} & 0 \\ O & W_{S H} & I\end{array}\right]$.

In this paper, we adopt the specification of WTMs presented by Yassine et al. (2003). Let $\Omega_{L}$ and $\Omega_{S}$ denote the DSMs within the local and system teams, respectively. For each $\sigma=$ $L, S$, the diagonal elements of $\Omega_{\sigma}$ give work completion coefficients, while the off-diagonal elements $\left(\Omega_{\sigma, i j}\right.$ with $\left.i \neq j\right)$ denote the amount of rework created for the task $i$ per unit of work done on the task $j$. Also, for describing the dependency between the local and system teams, inter-component dependency matrices (IDMs) are introduced. Let $\Omega_{L S}$ denote the IDM, whose element $\Omega_{L S, i j}$ represents the amount of rework created for system task $i$ per unit of work done on local task $j$. The IDM $\Omega_{S L}$ is understood in the same manner. Then, the WTM within the local team is given by

$W_{L, i j}= \begin{cases}1-\Omega_{L, i i}, & \text { if } i=j, \\ \Omega_{L, i j} \Omega_{L, j j}, & \text { if } i \neq j .\end{cases}$

The system WTM $W_{S}$ is computed in the same manner. Similarly, the IDMs are given by $W_{L S, i j}=\Omega_{L S, i j} \Omega_{L, j j}$ and $W_{S L, i j}=\Omega_{S L, i j} \Omega_{S, j j}$ for all $i$ and $j$. Finally, they let $W_{S H}=W_{S L}$.

Now, let $\tau_{0}, \tau_{1}, \ldots$ denote the times at which feedback from the system team to the local team occurs (see Fig. 1 for a schematic picture). Let $\Delta \tau_{\ell}=\tau_{\ell+1}-\tau_{\ell}$ denote the interval between feedbacks. In Yassine et al. (2003), it was assumed that the interval $\Delta \tau_{\ell}$ is constant. However, in practical PD processes, it is common that the interval of feedbacks 
Part III: Project Management

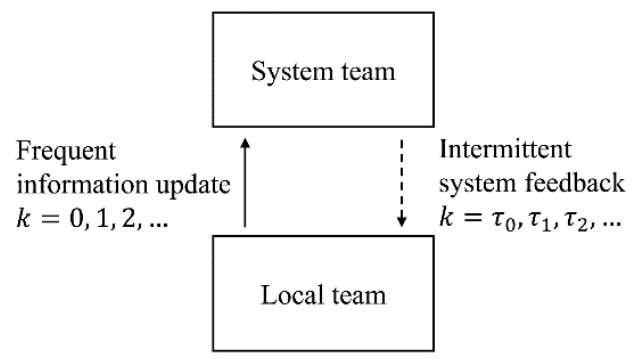

Figure 1 Asynchronous and aperiodic information exchange between the system and local teams.

fluctuates due to both intrinsic and exogenous factors. For this reason, we consider a generalized work transformation model where the interval experiences stochastic fluctuations. Let $h_{\min }$ and $h_{\max }$ denote the minimum and maximum length of interval, i.e., we assume that $0<h_{\min } \leq \Delta \tau_{\ell} \leq h_{\max }$. Let us assume that the random intervals $\Delta \tau_{\ell}$ are independent and identically distributed with the probability $\left(\Delta \tau_{\ell}=h\right)=p_{h}$ for $h=$ $1,2, \ldots$, which could be estimated by the PD manager from the past history of project management data. We remark that, under this formulation, we can no longer depend on the Floquet theory on periodic linear dynamical systems that was used in Yassine et al., (2003) due to the randomness in $\Delta \tau_{\ell}$.

\section{Feasibility Analysis}

In this section, we analyze the feasibility of the PD process described in the last section. We start our analysis by reviewing the results presented by Yassine et al. (2003), in which they have considered the case of periodic feedbacks from the system team to the local team. Let the period of the feedback be denoted by $T>0$. In their analysis, an important role is played by the so-called monodromy matrix $A_{2}^{T-1} A_{1}$, which represents the work transformation between feedback epochs, i.e., the transitions of the sub-sampled state variables $(0), x(T), x(2 T), \ldots$. However, in our case where the interval of feedbacks is stochastically modeled, the monodromy matrix equals $A_{2}^{\Delta \tau_{\ell}-1} A_{1}$, which is a random matrix. Due to this stochasticity of the monodromy matrix, we can no longer apply the Floquet theory to analyze the PD process. However, we can still compute the mathematical expectation of the monodromy matrix as

$\mathcal{M}=\left(p_{h_{\min }} A_{2}^{h_{\min }-1}+\cdots+p_{h_{\max }} A_{2}^{h_{\max }-1}\right) A_{1}$,

where $h_{\min }$ and $h_{\max }$ are the minimum and maximum of the interval of two subsequent feedbacks. By utilizing the theory of switched linear systems (Ogura and Martin, 2015) in the context of systems and control theory, we can, in fact, show that the eigenvalues of the matrix $\mathcal{M}$ completely determines the feasibility of the PD process, as stated in the following theorem: 
Theorem 1 The expected amounts of unfinished works, i.e., $E[L(k)]$ and $E[S(k)]$, converge to 0 as $k$ tends to $\infty$ if and only if the magnitudes of the eigenvalues of $\mathcal{M}$ are less than one.

The proof of this theorem can be found in (Ogura et al., 2019). By Theorem 1, the maximum magnitude of the eigenvalues of the matrix $\mathcal{M}$, i.e., the spectral radius $\rho(\mathcal{M})$, determines the feasibility of the PD project. For this reason, we call the spectral radius the feasibility index of the PD project.

\section{Optimizing Dependencies}

In this section, we study the problem of tuning the dependencies between distinct design components or teams to improve a nominal, possibly infeasible, PD process. We specifically consider the following PD optimization problem. Suppose that we can use resources for decreasing the dependencies. Assuming that the resources have an associated cost and that we are given a fixed budget, how should we distribute our resource throughout the local and system tasks to accelerate the PD process?

Let us focus on tuning the values of the inter-component or inter-team dependencies

$\left\{\Omega_{\sigma, i j}:(\sigma \in\{L S, S L\})\right.$ or $(i \neq j$ and $\left.\sigma \in\{L, S\})\right\}$.

We assume that there is a cost associated with tuning the strength of dependencies. Let $f_{L, i j}\left(\Psi_{L, i j}\right)$ denote the cost of tuning the nominal dependency $\Omega_{L, i j}$ to $\Psi_{L, i j}$. In other words, if we want to set the dependency of the $i$ th local component on the $j$ th local component to be $\Psi_{L, i j}$, we need to pay $f_{L, i j}\left(\Psi_{L, i j}\right)$ monetary units. Since we do not need to consider improving the dependencies that are originally zero, the total cost for the improvement of the DSM $\Omega_{L}$ equals $C_{L}=\sum_{(i, j): \Omega_{L, i j} \neq 0, i \neq j} f_{L, i j}\left(\Psi_{L, i j}\right)$. Similarly, we introduce the cost functions $f_{L S, i j}\left(\Psi_{L S, i j}\right), f_{S L, i j}\left(\Psi_{S L, i j}\right)$, and $f_{S, i j}\left(\Psi_{S, i j}\right)$ for tuning the nominal DSM $\Omega_{S}$ and IDMs $\Omega_{L S}$ and $\Omega_{S L}$, respectively. Then, the total cost for the improvement of the DSM and IDMs are given by $C_{S}=\sum_{(i, j): \Omega_{S, i j} \neq 0, i \neq j} f_{S, i j}\left(\Psi_{S, i j}\right), C_{L S}=\sum_{(i, j): \Omega_{L S, i j} \neq 0} f_{L S, i j}\left(\Psi_{L S, i j}\right)$ and $C_{S L}=\sum_{(i, j): \Omega_{S L, i j} \neq 0} f_{S L, i j}\left(\Psi_{S L, i j}\right)$. Thus, the total cost for the improvement of the entire development process equals

$C=C_{L}+C_{S}+C_{L S}+C_{S L}$.

We impose the following two natural restrictions on the new DSMs and IDMs. First, the new matrices must be "better" than the nominal ones. In other words, we impose the following inequalities

$\Psi_{\sigma, i j} \leq \Omega_{\sigma, i j}$,

where $\sigma$ denotes any one of the strings $L, S, L S$, and $S L$. Secondly, we assume that there is a certain management limitation in improving the values of the matrices, which we model by the inequality

$\Psi_{\sigma, i j} \geq \epsilon \Omega_{\sigma, i j}$,

where $\epsilon \in(0,1)$ is a constant dependent on projects. This inequality implies that the possible improvement of the values of the matrices is at most $100(1-\epsilon) \%$. 


\section{Part III: Project Management}

From our feasibility analysis in the last section, we know that the smaller the feasibility index, the faster the amounts of unfinished work decrease. For this reason, we formulate our PD project optimization problem as follows:

Problem (Budget-constrained dependency optimization) Given cost functions $f_{\sigma, i j}$, a constant $\epsilon>0$, and a budget $B>0$, find the new DSMs and IDMs $\Psi_{\sigma}$ that minimize the feasibility index $\rho(\mathcal{M})$ of the PD project while satisfying the budget constraint on the cost $C \leq B$ as well as the constraints (5) and (6).

The budget-constrained dependency optimization is not trivial to solve because the problems involve the spectral radius of the matrix $\mathcal{M}$ having the complicated expression (2). In this section, we show that the optimization problem can be efficiently solved if the cost functions $f_{\sigma, i j}$ belong to a wide class of functions called posynomials (see, e.g., (Boyd et al., 2007)), which we review below. Let $f(X)$ be a real function defined for a positive scalar variable $X>0$. We say that $f$ is a monomial if there exist $c>0$ and a real number $a$ such that $f(X)=c X^{a}$. We say that $f$ is a posynomial if $f$ is a sum of monomials. For example, $f(X)=X^{-2}+2+3 X^{1.5}$ is a posynomial because $X^{-2}, 2$, and $3 X^{1.5}$ are monomials.

We assume that, for each $\sigma, i$, and $j$, there exists a posynomial $f_{\sigma, i j}^{+}$such that the cost function $f_{\sigma, i j}$ is of the form $f_{\sigma, i j}\left(\Psi_{\sigma, i j}\right)=f_{\sigma, i j}^{+}\left(\Psi_{\sigma, i j}\right)-f_{\sigma, i j}^{+}\left(\Omega_{\sigma, i j}\right)$. The essential part of the cost function is the first term $f_{\sigma, i j}^{+}\left(\Psi_{\sigma, i j}\right)$, while the second term $-f_{\sigma, i j}^{+}\left(\Omega_{\sigma, i j}\right)$ is for normalizing the cost function as $f_{\sigma, i j}\left(\Omega_{\sigma, i j}\right)=0$, i.e., the zero investment yields the nominal interdependency matrix. Corresponding to the decomposition, let us define $C_{L}^{+}=$ $\sum_{(i, j): \Omega_{L, i j} \neq 0, i \neq j} f_{L, i j}^{+}\left(\Psi_{L, i j}\right), \quad C_{S}^{+}=\sum_{(i, j): \Omega_{S, i j} \neq 0, i \neq j} f_{S, i j}^{+}\left(\Psi_{S, i j}\right), \quad C_{L S}^{+}=\sum_{(i, j): \Omega_{L S, i j} \neq 0}$ $f_{L S, i j}^{+}\left(\Psi_{L S, i j}\right), \quad C_{S L}^{+}=\sum_{(i, j): \Omega_{S L, i j} \neq 0} f_{S L, i j}^{+}\left(\Psi_{S L, i j}\right)$ and $C_{L}^{-}=\sum_{(i, j): \Omega_{L, i j} \neq 0, i \neq j} f_{L, i j}^{+}\left(\Omega_{L, i j}\right)$, $C_{S}^{-}=\sum_{(i, j): \Omega_{S, i j} \neq 0, i \neq j} f_{S, i j}^{+}\left(\Omega_{S, i j}\right), \quad C_{L S}^{-}=\sum_{(i, j): \Omega_{L S, i j} \neq 0} f_{L S, i j}^{+}\left(\Omega_{L S, i j}\right), \quad C_{S L}^{-}=\sum_{(i, j): \Omega_{S L, i j} \neq 0}$ $f_{S L, i j}^{+}\left(\Omega_{S L, i j}\right)$. Let $C^{+}=C_{L}^{+}+C_{S}^{+}+C_{L S}^{+}+C_{S L}^{+}$and $C^{-}=C_{L}^{-}+C_{S}^{-}+C_{L S}^{-}+C_{S L}^{-}$. Then, the total cost (5) is rewritten as $C=C^{+}-C^{-}$.

We are now ready to state our second main theoretical result of this paper.

Theorem 2 Let $\Xi_{\sigma, i j}^{\star}$ be the solution of the optimization problem

$$
\begin{array}{ll}
\operatorname{minimize}_{\Xi_{\sigma, i j}} & \log \rho(\mathcal{M}) \\
\text { subject to } & \Xi_{\sigma, i j} \leq \log \Omega_{\sigma, i j}, \\
& \Xi_{\sigma, i j} \geq \log \epsilon+\log \Omega_{\sigma, i j}, \\
& \log C^{+} \leq \log \left(B+C^{-}\right) .
\end{array}
$$

Then, the DSMs and IDMs defined by

$$
\Psi_{\sigma, i j}^{\star}= \begin{cases}0, & \text { if } \Omega_{\sigma, i j}=0, \\ \exp \left(\Xi_{\sigma, i j}^{\star}\right), & \text { otherwise, }\end{cases}
$$



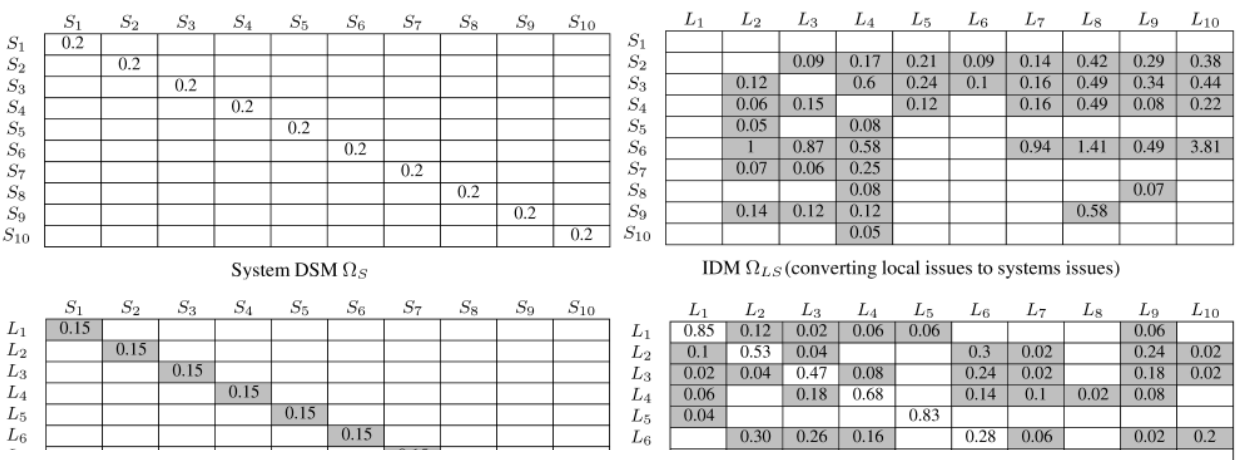

Figure 2 The nominal DSMs and IDMs of the automotive appearance design. The intercomponent and inter-team dependencies (3) that can be weakened by the manager are highlighted with the gray color.

solve the budget-constrained dependency optimization problem. Moreover, if the cost functions $f_{\sigma, i j}$ are posynomial, then this optimization problem is convex.

The proof of this theorem can be found in (Ogura et al., 2019). Theorem 2 serves as an analytical decision support tool for PD managers. Specifically, the theorem allows PD managers to efficiently solve the budget-constrained dependency optimization problem by using off-the-shelve software for convex optimization.

\section{Case Study: Automotive Appearance Design}

The automotive appearance design process reported in (McDaniel, 1996) and further investigated in (Yassine et al., 2003) is a part of an automobile PD process and refers to the process of designing all interior and exterior automobile surfaces. Yassine et al. (2003) focused on the following pair of the system and local teams; the engineering (local) team responsible for the feasibility of designs, and the styling (system) team responsible for the appearance of the vehicle. Information exchanges occur not only on the cross-functional level but also within functional groups working with specific tasks on appearance design. During the project period, there occur two different types of information exchanges between the teams. One is a weekly feasibility meeting, where the engineering team feedbacks to the styling team on infeasible design conditions. The other ones are in terms of CAD data from the styling team to the engineering team and are scheduled to be roughly six-week intervals. In this paper, we consider the situation where the schedule of the meeting can be either brought forward or postponed at most two weeks due to random and unexpected circumstances. To realize this situation, we set the minimum and the maximum interval of feedbacks as $h_{\min }=4$ and $h_{\max }=8$. 
Part III: Project Management

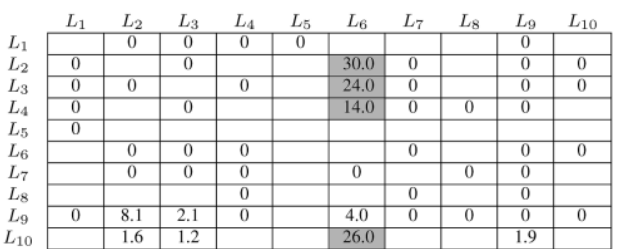

(a) $f_{L, i j}\left(\Psi_{L, i j}\right)$

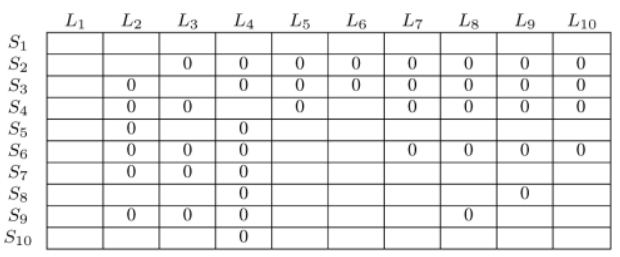

(b) $f_{L S, i j}\left(\Psi_{L S, i j}\right)$

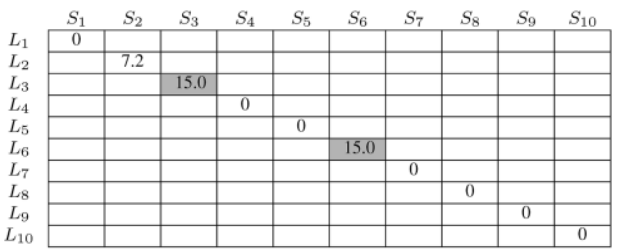

(c) $f_{S L, i j}\left(\Psi_{S L, i j}\right)$

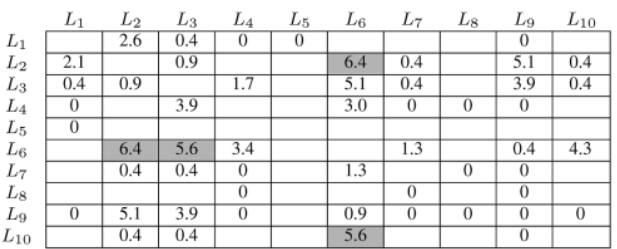

(a) $f_{L, i j}\left(\Psi_{L, i j}\right)$

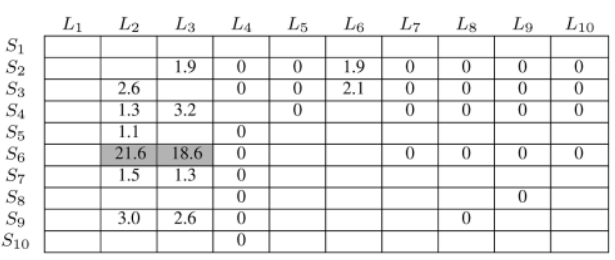

(b) $f_{L S, i j}\left(\Psi_{L S, i j}\right)$

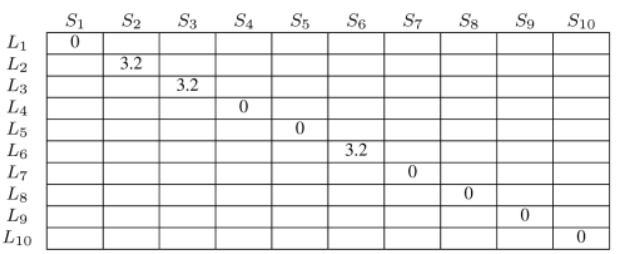

(c) $f_{S L, i j}\left(\Psi_{S L, i j}\right)$

Figure 3 Proposed (left column) and conventional (right column) investments on dependencies (multiplied by 100). The top six investments are highlighted with gray color.

In the nominal DSMs and IDMs shown in Fig. 2, we identify 104 dependencies of the form (3). Since the DSM $\Omega_{S}$ is diagonal and, therefore, does not contain dependency terms, we do not consider tuning the values of $\Omega_{S}$ in this case study.

We consider the following requirements on the cost functions in this case study: 1) the cost function $f_{\sigma, i j}$ is decreasing, that is, the more we invest, the weaker dependencies become; 2) to achieve the full improvement and set the dependency to $\epsilon \Omega_{\sigma, i j}$, we need to pay the $\operatorname{cost} \Omega_{\sigma, i j}$, that is, $f_{\sigma, i j}\left(\epsilon \Omega_{\sigma, i j}\right)=\Omega_{\sigma, i j}$. The second requirement in particular implies that the cost for the full improvement is proportional to the strength of the nominal dependencies. In order to realize this situation, we use the following cost function: $f_{\sigma, i j}\left(\Psi_{\sigma, i j}\right)=c_{\sigma, i j}\left(\frac{1}{\left(\Psi_{\sigma, i j}\right)^{p}}-\frac{1}{\left(\Omega_{\sigma, i j}\right)^{p}}\right)$, where $p$ is a parameter that should be appropriately set by the manager, and the constant $c_{\sigma, i j}$ is uniquely determined to satisfy $f_{\sigma, i j}\left(\epsilon \Omega_{\sigma, i j}\right)=$ $\Omega_{\sigma, i j}$. Also, we use a positive $p$ to satisfy the first requirement.

To set the baseline strategy for resource distribution, we recall the strategy that was found to be most effective among the other strategies that were proposed by Yassine et al. (2003).

In this reference, the tasks $L_{2}, L_{3}$, and $L_{6}$ were identified as "complex local components". Then, the authors proposed that the project manager should focus on weakening the 


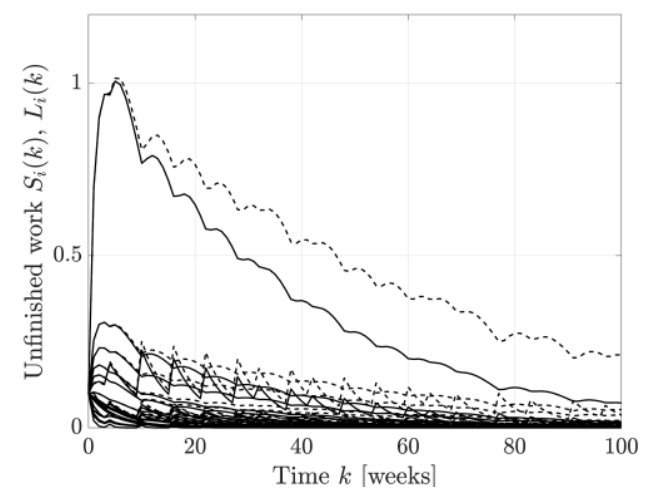

Figure 4 Amount of unfinished work for each design component and teams. Solid lines: proposed strategy. Dashed lines: baseline strategy.

dependencies between these three local tasks and other tasks. Therefore, in terms of the DSMs and IDMs, it was recommended that we weaken the following dependencies: $\Omega_{L, i 2}$, $\Omega_{L, i 3}, \Omega_{L, i 6}, \Omega_{L, 2 j}, \Omega_{L, 3 j}, \Omega_{L, 6 j}, \Omega_{L S, i 2}, \Omega_{L S, i 3}, \Omega_{L S, i 6}, \Omega_{S L, 2 j}, \Omega_{S L, 3 j}$, and $\Omega_{S L, 6 j}$ for $i, j=$ $1, \ldots, 10$. We choose to implement this recommendation in the following manner. In our implementation, we distribute the whole resource to the dependencies in such a way that the distributed resources are proportional to the strength of the nominal dependencies. Hence, in the baseline strategy, we distribute no resources to the dependencies that are not listed above.

For simplicity of illustration, we fix the probabilities of feedback intervals as $p_{1}=p_{2}=$ $p_{3}=0, p_{4}=p_{5}=1 / 8, p_{6}=1 / 2$, and $p_{7}=p_{8}=1 / 8$. We set $\epsilon=0.85$ (i.e., a full investment eliminates $15 \%$ of the nominal dependency) and solve the budget-constrained dependency optimization problem with the budget $B=1.5$. Using the baseline and proposed strategies, we obtain the two sets of resource distributions for weakening the strength of dependencies. The proposed strategy achieves the better performance $\rho(\mathcal{M})=$ 0.8484 compared with the baseline strategy $\rho(\mathcal{M})=0.9079$ (i.e., a smaller feasibility index is achieved by the proposed strategy). We show the obtained investments in Fig. 3. A remarkable difference between the proposed and baseline strategies can be found in the investments on $\Omega_{L S}$; although the proposed strategy invests no resource on $\Omega_{L S}$, the baseline strategy spends more than the half of the budget in its improvement. Let us then compare the amounts of reductions in project completion times. In Fig. 4, we show the trajectories of the amount of unfinished work when the proposed and baseline strategies are applied. We see that the proposed strategy achieves a faster decay in the amount of unfinished work.

\section{Conclusion}

In this paper, we have presented an analytical framework for optimizing the feasibility of PD projects having a time-varying architecture. We have focused on the specific but widely-observed situation in which system and local team asynchronously interact for development. By using a result from the systems and control theory, we first have derived 


\section{Part III: Project Management}

the feasibility index of the project for determining the long-term feasibility of the project. Building on this analysis result, we have shown that the optimal resource distribution solving the budget-constrained and performance-constrained dependency optimization problems can be efficiently found by solving convex optimization problems. The effectiveness of the framework has been illustrated by the case study taking an automobile $\mathrm{PD}$ project as an example.

\section{References}

Adler, P., Mandelbaum, A., Nguyen, V., Schwerer, E., 1995. Project to process management: empirically-based framework for analyzing product development time. Management Science 41, 458-484.

Baldwin, C., Clark, K., 2000. Design Rules: The Power of Modularity. MIT Press.

Boyd, S., Kim, S.-J., Vandenberghe, L., Hassibi, A., 2007. A tutorial on geometric programming. Optimization and Engineering 8, 67-127.

Boyd, S., Vandenberghe, L., 2004. Convex Optimization. Cambridge University Press.

Browning, T.R., Ramasesh, R.V., 2007. A Survey of activity network-based process models for managing product development projects. Production and Operations Management 16, 217 240 .

Hartmann, S., Briskorn, D., 2010. A survey of variants and extensions of the resource-constrained project scheduling problem. European Journal of Operational Research 207, 1-14.

Lee, H., Padmanabhan, V., Whang, S., 1997. Information distortion in a supply chain: the bullwhip effect. Management Science 43, 546-558.

Loch, C., Terwiesch, C., 1999. Accelerating the process of engineering change orders: capacity and congestion effects. Journal of Product Innovation Management 16, 145-159.

McDaniel, C.D., 1996. A linear system framework for analyzing the automotive appearance design process ( $\mathrm{PhD}$ thesis). Massachusetts Institute of Technology.

Ogura, M., Harada, J., Kishida, M., Yassine, A., 2019. Resource optimization of product development projects with time-varying dependency structure. Research in Engineering Design 30, 435-452.

Ogura, M., Martin, C.F., 2015. Stability analysis of linear systems subject to regenerative switchings. Systems \& Control Letters 75, 94-100.

PMI, 2013. A Guide to the Project Management Body of Knowledge. Project Management Institute.

Yassine, A., Joglekar, N., Braha, D., Eppinger, S., Whitney, D., 2003. Information hiding in product development: The design churn effect. Research in Engineering Design 14, 145-161.

Contact: M. Ogura, Division of Information Science, Nara Institute of Science and Technology, 8916-5 Takayama, Ikoma, 630-0192 Nara, Japan, +81 743725353, oguram@is.naist.jp 